\title{
Tympanolith in the Middle Ear
}

\author{
Ahilasamy Nagalingeswaran ${ }^{1}$ Rajendran Dinesh Kumar² \\ ${ }^{1}$ Ahilasamy ENT Centre, Chennai, Tamil Nadu, India \\ ${ }^{2}$ Department of ENT and Head-Neck Surgery, P. D. Hinduja Sindhi \\ Hospital, Bengaluru, Karnataka, India
}

Address for correspondence Rajendran Dinesh Kumar, MBBS, MS ENT, Department of ENT and Head-Neck Surgery, P. D. Hinduja Sindhi Hospital, Bengaluru 560027, Karnataka, India (e-mail: dinuraj1186@gmail.com).

\begin{abstract}
Keywords

- tympanolith

- middle ear cavity

- endoscopic

Background A tympanolith is a calcified body within the middle ear cavity, arising as a result of calcification as a possible nidus (extrinsic or intrinsic).

Case Report A male patient with a known history of diabetes presented with painless, non-foul smelling, chronically discharging right ear more than 20 years duration with an associated hearing loss. Routine investigations were within normal limits, pure tone audiometry findings were suggestive of profound hearing loss in the affected ear. Otoendoscopy revealed a tympanolith, which was removed, following which a moderate size central perforation with an edematous middle ear mucosa was noted.

Discussion Tympanolith is a rare condition which is known to occur in a chronically discharging ear, it's usually hard, gritty with a smooth surface.

Conclusion In chronically discharging ears with a calcified mass lesion, tympanolith should be considered as differential diagnosis.
\end{abstract}

\section{Introduction}

A tympanolith is a calcified mass within the tympanic cavity. The origin of the nidus of calcification may be extrinsic (foreign body) or intrinsic (stagnant mucus and inspissated purulent dried discharge). These masses are rare but not unusual. Most of them are asymptomatic in small tympanolith. Larger ones may present with symptoms of ear discharge and hard of hearing, for which oto-endoscopic complete removal and its chemical assessment are required.

\section{Case Report}

Our first case was a 70-year-old man, diabetic with painless, non-foul-smelling, chronically discharging right ear for 20 years with hard of hearing since 10 years. There was no prior history of foreign-body insertion or hearing aid usage.On oto-endoscopic examination, mucopurulent discharge was noted amidst small posterior canal wall swelling ( - Fig. 1A). Following suction clearance of the mucopurulent discharge, a smooth fluctuating posterior ear canal wall swelling with a blackish discharge through the tympanic perforation was noted $(-$ Fig. 1B).
The patient was routinely investigated for preanesthetic evaluation and taken for surgery under local anesthesia. Routine investigation workup was within normal limits. Diabetes euglycemic status and pure tone audiometry were suggestive of bilateral mixed hearing loss with profound hearing loss in the affected ear.

During oto-endoscopy, it was found to be tympanolith, which was successfully removed en masse by angled probe from middle ear cavity without injuring surrounding mucosa or external auditory canal skin (-Fig. 1C, D). After thorough normal saline wash of the external canal and tympanic cavity, local anesthesia (1\% Xylocaine with 1:80,000 adrenaline) was infiltrated in the external auditory canal. Posterior canal wall swelling was soft and fluctuant. On incision, a purulent secretion drained out following which thorough normal saline wash of the external canal and tympanic cavity was repeated.

Because of chronic inflammation by the tympanolith occupying middle ear cavity, the inner aspect of the external auditory canal was considerably narrow, edematous compared with the outer aspect of the external auditory canal. 

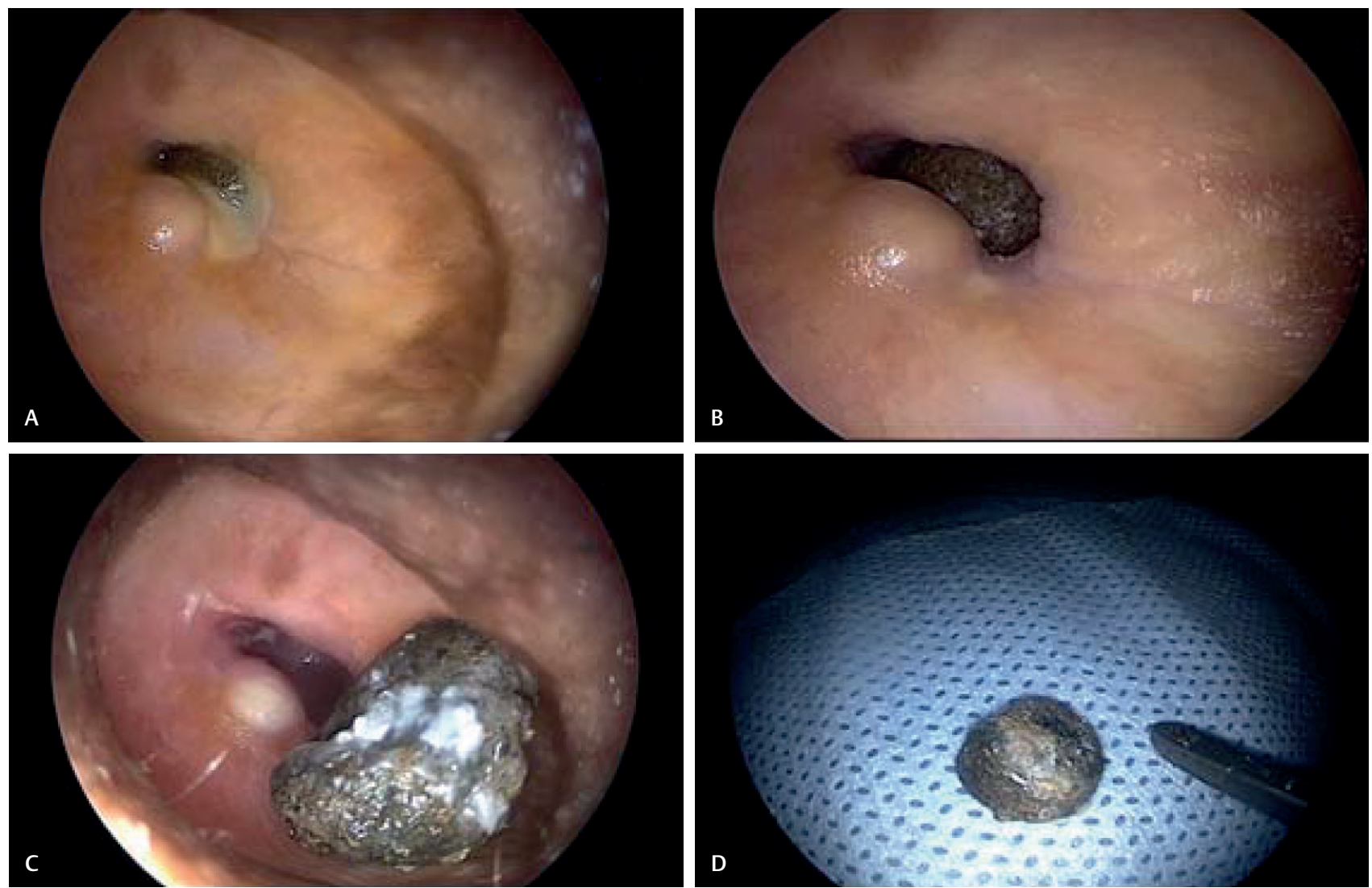

Fig. 1 Tympanolith in the middle ear. (A) Oto-endoscopy of right ear. (B) Right ear oto-endoscopic image after suction clearance. (C) Tympanolith successfully removed en masse by angled probe from middle ear cavity; posterior canal wall swelling with moderate-size central perforation noted. (D) Tympanolith.

Moderate-size central perforation of the tympanic membrane was noted with an edematous middle ear mucosa. Conventional endoscopic tympanoplasty with homologous septal cartilage graft was done under local anesthesia.

The black, hard gritty smooth surface material was sent for stone analysis by Fourier transform infrared spectrometry (FTIR) method that showed the stone size to be $7 \times 6 \mathrm{~mm}$, weighing $0.298 \mathrm{~g}$ and composed mainly of calcium oxalate dehydrate $50 \%$, calcium oxalate monohydrate $30 \%$, and sodium urate $20 \%$, suggestive of calculi body origin ( - Fig. 2 ). As in this case, the long-standing chronic stagnant ear discharge and mucus accounted to formation of black gritty smooth pebble-like mass, mimicking foreign body on first look oto-endoscopic examination.

The patient was put on a course of antibiotics, analgesics, and antihistamines postoperatively, and followed up regularly till date. It is asymptomatic with a healed drum on follow-up ( - Video 1).

\section{Discussion}

Tympanolith is a rare condition known to occur in the middle ear cavity from long-standing chronic suppurative otitis media with central perforation similar to a sinolith. The origin of the nidus of calcification may be extrinsic (foreign body) or intrinsic (stagnant mucus and inspissated purulent dried discharge).
Tympanolith conditions are hard, gritty, and smooth surface. To our knowledge, one case has been reported in literature. ${ }^{1}$ This case is presented for its rarity due to chronic inflammation and ear discharge that may have dried up from frequent middle ear mucosal secretions, acting as an endogenous source of nidus allowing calcium salts to be deposited around the nucleus forming a tympanolith.

A hypothetical metabolic disorder might have led to precipitation of calcium and magnesium salts in the chronically inflamed middle ear cleft. However, evidence for this was not found clinically or biochemically. ${ }^{1}$

Only one publication on this subject was found in the current literature by Gapany-Gapanavicius et al. ${ }^{1}$ No available handbook or other reference text on otology presents mention of calculus formation in the middle ear cavity. ${ }^{2-10} \mathrm{An}$ attempt was made to exclude all possible factors that might have led to formation of calculi in this case. It can be considered only as a concomitant symptom, a healing reaction of the suppurative process in the middle ear cleft.

\section{Video 1}

Tympanolith in middle ear. Online content including video sequences viewable at: https://www.thiemeconnect.com/ products/ejournals/html/doi/10.1055/s-0037-1612642. 


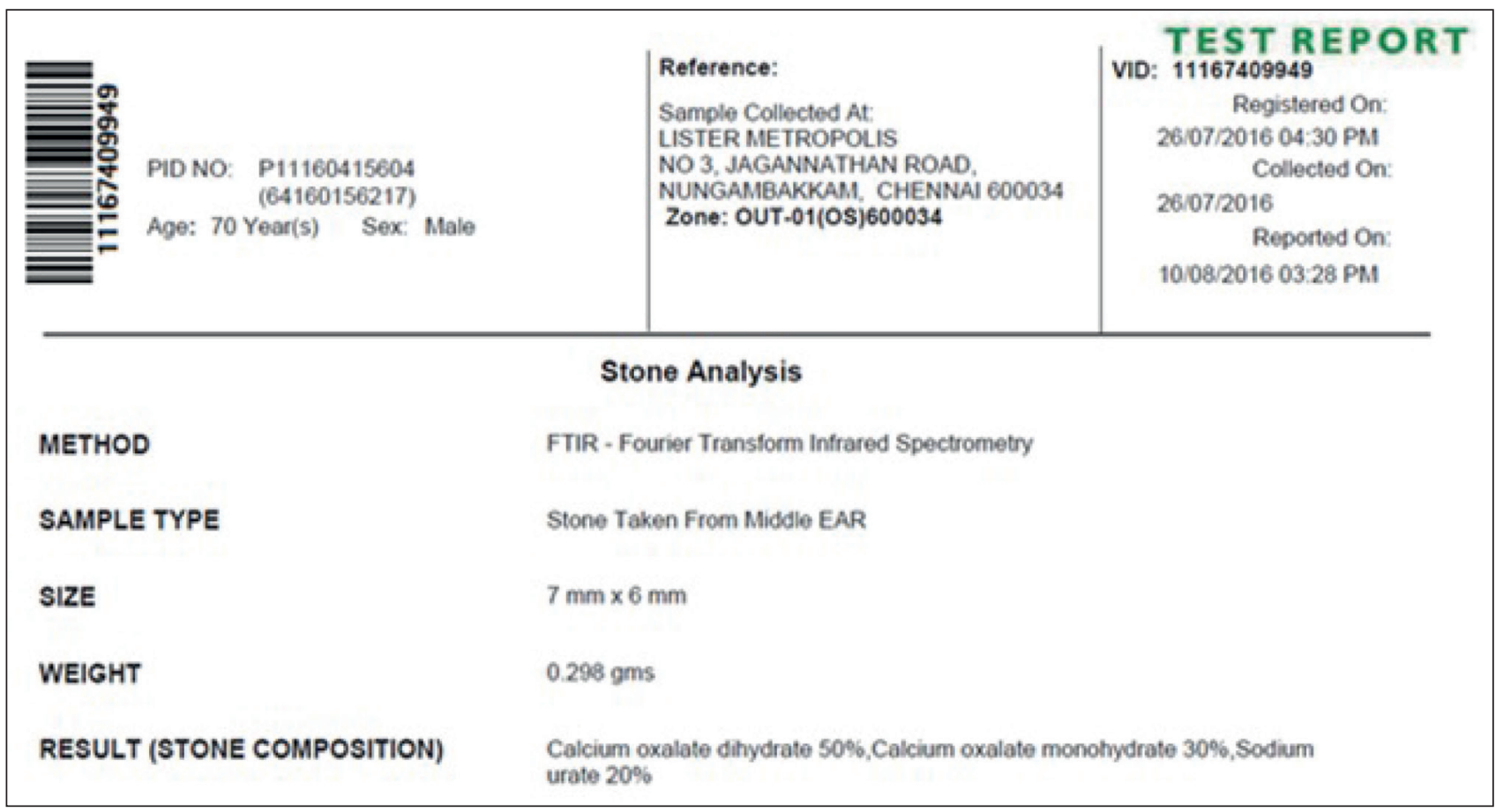

Fig. 2 Fourier transform infrared spectrometry report of the mass from the right middle ear cavity.

The possibilities of foreign bodies, general and local metabolic disorders, and calcified tuberculous lesions were taken into consideration. Foreign bodies could be excluded as a causative agent and nucleus for calcium precipitation and calculus formation, because no traces of foreign bodies of any kind were detected during thorough oto-endoscopic examination of the middle ear cavity.

\section{Conclusion}

Tympanolith should also be considered as differential diagnosis in chronically discharging ears. Most of the masses are asymptomatic in small tympanolith. Larger ones may present with symptoms of ear discharge and hard of hearing, for which oto-endoscopic complete removal and their chemical assessment are required.

\section{Funding}

None.

\section{Conflict of Interest \\ None.}

\section{Authors' Contributions}

Ahilasamy Nagalingeswaran performed surgery and prepared the manuscript and patient pre- and postoperative management and follow-up.

Rajendran Dinesh Kumar prepared the manuscript, literature review, and review of manuscript.

\section{Informed Consent}

Informed consent was obtained from all participants included in the study.

\section{Ethical Approval}

All procedures performed in studies involving human participants were in accordance with the ethical standards of the institutional and/or national research committee and with the 1964 Helsinki declaration and its later amendments or comparable ethical standards.

\section{References}

1 Gapany-Gapanavicius B, Sela J, Levij IS. Chronic tympanomastoiditis with formation of calculi. Ann Otol Rhinol Laryngol 1977;86(3 Pt 1):386-391

2 Schuknecht HF. Pathology of the Ear. Cambridge, MA: Harvard University Press; 1974:229-233

3 Friedmann I. Pathology at the Ear. London, UK: Blackwell Scientific; 1974:71-76

4 Manasse P. Handbuch der Pathologischen Anatomie des Menschlicheti Ohres. Wiesbaden, Germany: JF Bergmann; 1917

5 Denker A, Kahler O. Handbuch der Hals-Nasen-OhrenHeilkunde. Die Kronkheiten des Gehororgans. Band I-II-III. Berlin, Germany: J. Springer; 1926

6 Kopetzky SJ. Surgery of the Ear. New York, NY; Edinburgh, UK: T. Nelson and Sons; 1947

7 Lederer FL. Diseases of the Ear, Nose and Throat. Philadelphia, PA: F.A. Davies; 1953

8 Shambaugh GE. Surgery of the Ear. Philadelphia, PA; London, UK: W.B. Saunders; 1959

9 Voyachek VI, Preobrazhensky BS, eds. Mtiogotomrunje Rukovodstvo po Otorinolaringologii. Tom 2, Bolezni Ucha. Moskva, Russia: Medgiz; 1960 (Russian)

10 Ballenger JJ. Diseases of the Nose, Throat and Ear. 10th ed. Philadelphia, PA: Lea and Febiger; 1969 